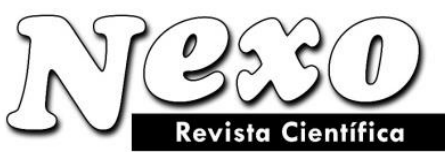

Vol. 33, No. 01, pp. 36-50/Junio 2020

\title{
EFECTO DE ACEITES DE Cocos nucifera Y Jatropha gossypiifolia SOBRE LA ESTABILIDAD DE ASFALTENOS
}

\section{EFFECT OF Cocos nucifera AND Jatropha gossypiifolia OILS ON THE ASPHALTENES STABILITY}

\author{
T.D. Marín Velásquez ${ }^{1, *}$, D.D.J. Arriojas Tocuyo ${ }^{2}$ \\ ${ }^{1}$ Universidad de Oriente. Departamento de Ingeniería de Petróleo. Maturín, Venezuela. \\ ${ }^{2}$ Innova Scientific. Departamento de Investigación. Lima, Perú. \\ tmarin@protonmail.com
}

(recibido/received: 14-noviembre-2019; aceptado/accepted: 31-marzo-2020)

\section{RESUMEN}

El objetivo fue evaluar los aceites de Cocos nucifera y Jatropha gossypiifolia como estabilizantes de asfaltenos en un petróleo crudo mediano del Campo El Furrial Estado Monagas, Venezuela. Se utilizaron dos muestras por cada aceite, una de las cuales se calentó a $140{ }^{\circ} \mathrm{C}$ por $24 \mathrm{~h}$. Se prepararon tres mezclas por cada aceite en diésel $(30,40,50 \%)$. Se aplicaron $8 \mu \mathrm{l}$ de cada mezcla a $10 \mathrm{ml}$ de petróleo y se obtuvo el umbral de floculación (UF) de asfaltenos como los ml de n-heptano aplicados para observar agregados en un microscopio óptico y el punto de dispersión (PD) como los ml de xileno para solubilizar los agregados de asfaltenos formados. El Índice de Inestabilidad (INE) representa la relación entre UF y PD. El diseño experimental fue factorial con una variable respuesta (INE) y 3 factores experimentales (Tipo de Aceite, Temperatura y Porcentaje de Aceite). Se utilizó el paquete Statgraphics Centurion XVII con $\alpha=0,05$. Los factores experimentales y sus relaciones influyen significativamente en la estabilidad de asfaltenos en el petróleo utilizado. Ambos aceites estabilizan los asfaltenos, siendo mejor su desempeño al ser calentados a $140{ }^{\circ} \mathrm{C}$ y el de $J$. gossypiifolia fue el más eficiente con $71,89 \%$.

Palabras claves: Asfaltenos; Floculación; Estabilidad; Dispersión; Cocos nucifera; Jatropha gossypiifolia.

\section{ABSTRACT}

The objective was to evaluate Cocos nucifera and Jatropha gossypiifolia oils as asphaltene stabilizers in a medium crude oil from Campo El Furrial Estado Monagas, Venezuela. Two samples were used for each oil, one of which was heated to $140{ }^{\circ} \mathrm{C}$ for 24 hours. Three mixtures were prepared for each diesel oil $(30,40$, and $50 \%$ ). Eight $\mu$ were applied from each mixture to $10 \mathrm{ml}$ of petroleum and the flocculation threshold (UF) of asphaltenes was obtained as the $\mathrm{ml}$ of $\mathrm{n}$-heptane applied to observe aggregates in an optical microscope and the dispersion point (PD) as the $\mathrm{ml}$ of xylene to solubilize the asphaltene aggregates formed. The Instability Index (INE) represents the relationship between UF and PD. The experimental design was factorial with a response variable (INE) and 3 experimental factors (Type of Oil, Temperature and Percentage of Oil). The package Statgraphics Centurion XVII was used with $\alpha=0.05$. Experimental factors and their relationships significantly influence the stability of asphaltenes in the oil used. Both oils stabilize 
the asphaltenes, their performance being better when heated to $140{ }^{\circ} \mathrm{C}$ and that of $J$. gossypiifolia was the most efficient with $71.89 \%$.

Keywords: Asphaltenes; Flocculation; Stability; Dispersion; Cocos nucifera; Jatropha gossypiifolia.

\section{INTRODUCCIÓN}

El petróleo, se define como una mezcla compleja de hidrocarburos, que se encuentran en los tres estados de la materia (gas, líquido y sólido) que están en equilibrio termodinámico a las condiciones en las que el mismo se encuentre. La mayoría de los hidrocarburos componentes del petróleo son líquidos (saturados y aromáticos), sin embargo también están presentes componentes sólidos de alto peso molecular y estructura compleja, con diferentes proporciones de nitrógeno, azufre y oxígeno, conocidos con el nombre genérico de asfaltenos (Pineda-Flores y Mesta-Howard, 2001). A través del tiempo se han establecido diferentes criterios para definir a los asfaltenos, sin embargo se ha llegado a un consenso, al definirlos como la fracción del petróleo soluble en aromáticos como benceno, tolueno y xileno e insolubles en n-alcanos como pentano, hexano y heptano (Spiecker, Gawrys y Kilpatrick, 2003). Según Goual (2012) la definición de la clase de solubilidad de los asfaltenos genera una amplia distribución de las estructuras moleculares. Estas moléculas polidispersas consisten principalmente en aromáticos polinucleares (PNA) con diferentes proporciones de alifáticos y alicíclicos y pequeñas cantidades de heteroátomos (como oxígeno, nitrógeno, azufre) y metales pesados (como vanadio y níquel), que se encuentran en estructuras de porfirina.

Los asfaltenos al ser los componentes más pesados del petróleo, y por encontrarse en un estado pseudoestable, al someterse a ciertas condiciones desfavorables de presión, temperatura, composición química y tasa de corte, floculan, forman agregados y se precipitan afectando de manera negativa la recuperación de petróleo y las operaciones de tratamiento que se realizan en superficie (Sepúlveda, Bonilla y Medina, 2010). La condición de sólidos y su estabilidad ha generado teorías de como los asfaltenos se encuentran dentro del petróleo, siendo la más aceptada la teoría coloidal, la cual establece que los asfaltenos existen en el petróleo como partículas sólidas en suspensión coloidal estabilizada por resinas, adsorbidas sobre su superficie (Akbarzadeh et al., 2007; Mansoori, 2009). Al estar los asfaltenos suspendidos dentro de la matriz líquida del petróleo, su estabilidad depende de las condiciones termodinámicas a las que está sometido (Presión, Temperatura y Composición) por lo que, variaciones en estas condiciones pueden generar rompimiento del sistema coloidal, formación de agregados y separación de los mismos de la fase líquida, produciendo depósitos orgánicos que taponan u obstruyen tuberías y equipos de procesos (Safaie y Naza, 2014).

La estabilidad de los asfaltenos se mantiene a nivel de campo, mediante la adición de productos químicos dispersantes, formulados a base de resinas sintéticas y solventes aromáticos (Pereira et al., 2011; Saeedi y Hasan, 2017). Los componentes activos de estos productos dispersantes de asfaltenos son las resinas, las cuales poseen características surfactantes y cuyo principio de acción depende del tipo específico de la misma de su composición y los grupos funcionales que posean (Madh, Kharrat y Hamoule, 2017). El uso de compuestos dispersantes de asfaltenos, ha sido estudiado ampliamente, en búsqueda de mejores resultados en la estabilización y prevención de los problemas de taponamiento, producto del fenómeno de floculación y precipitación, que lleva a la formación de depósitos orgánicos sólidos, formados a su vez, también por resinas y ceras parafínicas (Mehrotra y Bidmus, 2004). Entre los estudios realizados sobre el efecto de compuestos estabilizantes de asfaltenos, Rezaee et al. (2013) se enfocaron en el estudio de un surfactante iónico ácido (3-(2-carboxibenzoil)-1-metil-1H-imidazol-cloruro de 3-ion), Ghaffar et al. (2013) también estudiaron un surfactante polimérico oxigenado tipo dodecilfenol formaldehído-b-poli (oxipropileno). En ambas investigaciones, los productos estudiados fueron compuestos sintéticos derivados de hidrocarburos del petróleo. 
Los productos dispersantes utilizados comúnmente, están formulados con componentes activos de origen sintético, los cuales ocasionan gastos considerables a la industria petrolera, además de ser un potencial riesgo ambiental por su baja degradabilidad al derramarse en el suelo o el agua. Por tal razón, se han venido realizando estudios con aceites y resinas de origen vegetal, como el aceite de coco (Bello, Manzano y Marín, 2015; Marín, 2019), el aceite de avellana (Safaie y Naza, 2014), aceite de Jatropha curcas (Marín, Marcano y Febres, 2016) y el aceite obtenido de la cáscara del Anacardiun occidentale (Afra et al., 2018). En todas las investigaciones se ha demostrado la aplicabilidad de los aceites vegetales y sus derivados como inhibidores o dispersantes de asfaltenos, con eficiencias mayores a $80 \%$. En la presente investigación se evalúan y comparan los aceites de coco (Cocos nucifera) y de Tua tua (Jatropha gossypiifolia) como componentes activos estabilizantes de asfaltenos, aplicados a una muestra de petróleo crudo mediano proveniente del Campo El Furrial en el Estado Monagas, Venezuela. Como se mencionó, el aceite de coco ha sido estudiado previamente y se ha comprobado de tiene potencial para ser aplicado como estabilizante de asfaltenos, sin embargo el aceite de la Tua tua, es una alternativa nueva. Este aceite representa una alternativa por ser no comestible (a diferencia del aceite de coco), además proviene de una planta que crece de manera silvestre en varios países de Latinoamérica y África (Félix-Silva et al., 2014). El uso de diferentes partes de esta planta ha sido estudiado ampliamente por su potencial medicinal (Oyedeji et al., 2018; Yaro et al., 2018; Islam, Islam \& Hossain, 2017; De Almeida et al., 2016), sin embargo existen pocos estudios de su aplicación de su aceite, sobre todo para producción de biocombustible.

El aceite de $J$. gossypiifolia ha sido poco estudiado, debido a que es más comercial el aceite de Jatropha curcas, por su potencial para producir biocombustible, mayor rendimiento de aceite, mayor distribución geográfica y las mejoras genéticas que se le han realizado. El aceite de J. gossypiifolia se ha estudiado previamente, solo a efectos de comparación, en composición y comportamiento con otras plantas de la especie Jatropha, por lo que la comparación de su desempeño respecto al aceite de coco es de suma importancia para establecer su potencial uso en la industria petrolera.

\section{MATERIALES Y MÉTODOS}

Este estudio se realizó en el Laboratorio de Procesamiento de Hidrocarburos del Departamento de Ingeniería de Petróleo de la Universidad de Oriente, Núcleo de Monagas, Venezuela.

Como materia prima se utilizaron, la pulpa de coco (C. nucifera) y semillas de Tua tua (J. gossypiifolia) obtenidos en la ciudad de Maturín, Estado Monagas, Venezuela y una muestra de petróleo del Campo El Furrial, Estado Monagas, Venezuela. El aceite de coco se extrajo por hidroextracción (artesanal) siguiendo el procedimiento descrito por Da Silva et al. (2017) y el aceite de Tua tua mediante extracción con n-hexano al 98\%, aplicando un procedimiento de extracción sólido-líquido con la ayuda de un equipo Soxhlet con balón de $500 \mathrm{ml}$ de capacidad, tomando como referencia a Lafont, Páez y Portacio (2011). A ambos aceites se les determinó la densidad por el método del picnómetro (ASTM D1298, 2012) y la viscosidad mediante un viscosímetro rotacional Brookfield (ASTM D2196, 2015). Se obtuvieron $50 \mathrm{ml}$ de cada aceite y se dividieron en dos proporciones de $25 \mathrm{ml}$ cada una, una de las cuales se introdujo en un horno de laboratorio y se sometió a calentamiento a una temperatura de $140{ }^{\circ} \mathrm{C}$ por $24 \mathrm{~h}$. Luego las mezclas fueron sacadas del horno y se dejaron enfriar hasta la temperatura ambiente del laboratorio $\left(26^{\circ} \mathrm{C}\right)$, se determinaron de nuevo las propiedades anteriormente mencionadas y se formularon tres mezclas de $20 \mathrm{ml}$ de cada aceite evaluado en diésel como solvente (30, 40 y 50\%). Los porcentajes de mezcla se establecieron a criterio de los investigadores. Al petróleo se le determinó la densidad o gravedad API por el método del hidrómetro, a partir de la norma ASTM D287 (2012) y la composición SARA (Saturados, Aromáticos, Resinas y Asfaltenos), aplicando la norma ASTM D2007 (2011).

Para la evaluación del efecto de cada una de las mezclas de aceite formuladas, se determinó el Índice de Inestabilidad de los asfaltenos, mediante el principio de la relación solubilidad/insolubilidad, aplicando los procedimientos descritos por Bello et al. (2015) y Marín (2019). El Índice de Inestabilidad (INE) se calcula

Nexo Revista Científica / Vol. 33, No. 01, pp. 36-50 / Junio 2020 
a partir del umbral de floculación (UF) y el Punto de Dispersión (PD) de los asfaltenos. El UF se define como el volumen de n-heptano utilizado para lograr que los asfaltenos presentes en el petróleo formen agregados visibles en un microscopio óptico y el PD se define como el volumen de Xileno utilizado para lograr que los agregados de asfaltenos formados, vuelvan a disolverse. El procedimiento se muestra en la figura 1 .

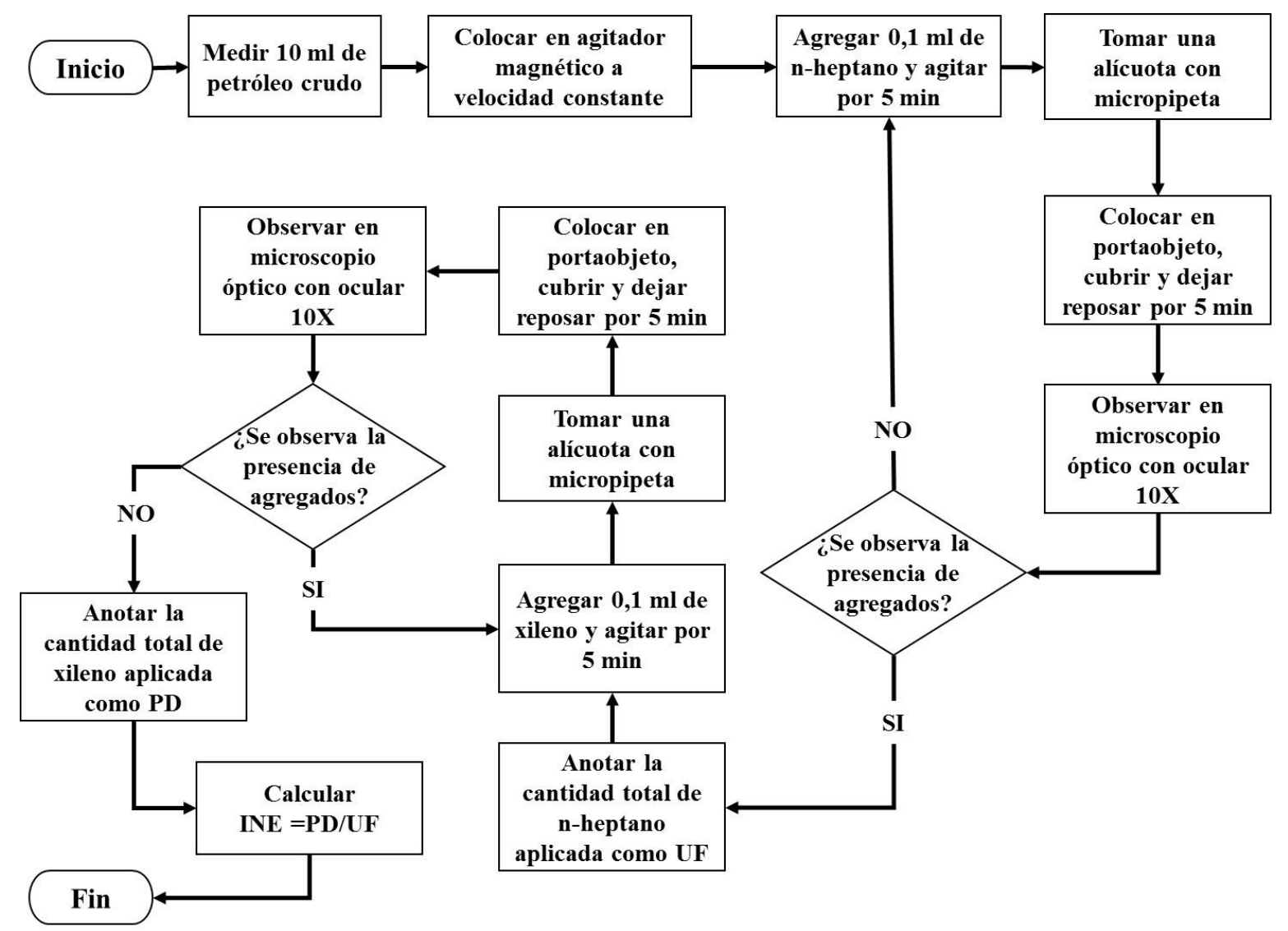

Figura 1. Procedimiento para determinar el UF y el PD.

Fuente: adaptado de (Marín, 2019)

El INE se calcula con la ecuación 1, la cual se muestra a continuación:

$$
I N E=\frac{P D}{U F}
$$

La eficiencia de cada tratamiento se calculó respecto al valor de INE patrón, es decir el del petróleo sin tratamiento, a partir de la ecuación 2.

$$
\% E F=\frac{I N E_{\text {Patrón }}-I N E_{\text {Tratamiento }}}{I N E_{\text {Patrón }}} \times 100
$$

El procedimiento descrito en la figura 1 corresponde a los UF y PD de la muestra de petróleo original, con lo que se obtuvo el INE original sin tratamiento (patrón) con el que se realiza la comparación y cálculo de eficiencia. El procedimiento se repite, aplicando $8 \mu \mathrm{l}$ de cada mezcla de aceite/diésel al petróleo, tomando como referencia lo indicado por Bello et al. (2015) y se calculó cada INE para cada mezcla, así como para $8 \mu \mathrm{l}$ de diésel puro. Los tratamientos aplicados se resumen en la tabla 1. 
T.D. Marín-Velásquez y D.D.J. Arriojas-Tocuyo

Tabla 1. Descripción de los tratamientos aplicados

\begin{tabular}{cccc}
\hline Tratamiento & Nomenclatura & $\begin{array}{c}\text { Temperatura } \\
\left({ }^{\circ} \mathrm{C}\right)\end{array}$ & $\begin{array}{c}\text { Porcentaje } \\
\text { de Aceite }\end{array}$ \\
\hline \multirow{2}{*}{ Diésel } & D26 & 26 & 0 \\
& D140 & 140 & 0 \\
Aceite de coco & AC2630 & 26 & 30 \\
& AC2640 & 26 & 40 \\
& AC2650 & 26 & 50 \\
& AC14030 & 140 & 30 \\
& AC14040 & 140 & 40 \\
& AC14050 & 140 & 50 \\
& AT2630 & 26 & 30 \\
& AT2640 & 26 & 40 \\
& AT2650 & 26 & 50 \\
& AT14030 & 140 & 30 \\
& AT14040 & 140 & 40 \\
& AT14050 & 140 & 50 \\
\hline & Fuente: Elaboración propia &
\end{tabular}

Se aplicó un diseño factorial con una variable respuesta (INE), tres factores experimentales (Diésel, AC y AT) y 14 tratamientos con tres réplicas cada uno. El diseño seleccionado tiene 48 ejecuciones, con 1 una muestra para ser tomada en cada ejecución. El modelo aplicado fue cúbico con 15 coeficientes. El análisis de validez interna del diseño experimental planteado fue analizado mediante el paquete estadístico Statgraphics Centurion XVI.I y se obtuvo que el error total tiene 36 grados de libertad. En general, es una buena idea tener al menos tres o cuatro grados de libertad en el error para que los efectos estimados sean estadísticamente significativos, lo que indica que los contrastes estadísticos tendrán una potencia significativa para el diseño experimental utilizado, demostrándose la validez del mismo.

Para el tratamiento estadístico de los resultados, se analizó inicialmente el supuesto de normalidad de los datos mediante la prueba de Kolmogorov-Smirnov modificada, con la cual se obtuvo un p-valor $=0,325$ lo que indica que no se puede descartar que los datos provengan de una distribución normal, por ser el resultado mayor del valor de significancia fijado $(\alpha=0,05)$. Por lo anterior se aplicó ANOVA multifactorial con la finalidad de observar la significancia del efecto de los factores experimentales sobre la variable respuesta (INE), además de una prueba de múltiples rangos por mínima diferencia significativa de Fisher para observar posibles diferencias entre los factores, respecto a la temperatura a la que fueron sometidos los aceites, los porcentajes utilizados y el tipo de aceite. Todos los análisis se realizaron con el paquete estadístico Statgraphics Centurion XVI.I.

\section{RESULTADOS}

En la tabla 2, se muestran las características obtenidas de los aceites evaluados luego de la aplicación de los procedimientos estandarizados.

Tabla 2. Propiedades de los aceites evaluados

\begin{tabular}{|c|c|c|c|c|c|}
\hline Aceite & Propiedad & Temperatura & Valor & Unidad & Norma \\
\hline \multirow{2}{*}{ Aceite de coco } & Densidad & $\begin{array}{c}26 \\
140\end{array}$ & $\begin{array}{l}0,92 \\
0,92\end{array}$ & $\mathrm{~g} / \mathrm{ml}$ & ASTM D1298 \\
\hline & Viscosidad & $\begin{array}{c}26 \\
140\end{array}$ & $\begin{array}{l}15,5 \\
65,6\end{array}$ & $\mathrm{cP}$ & ASTM D2196 \\
\hline \multirow{2}{*}{ Aceite de Tua tua } & Densidad & $\begin{array}{c}26 \\
140\end{array}$ & $\begin{array}{l}0,92 \\
0,92\end{array}$ & $\mathrm{~g} / \mathrm{ml}$ & ASTM D1298 \\
\hline & Viscosidad & $\begin{array}{c}26 \\
140\end{array}$ & $\begin{array}{l}54,3 \\
79,6\end{array}$ & $\mathrm{cP}$ & ASTM D2196 \\
\hline
\end{tabular}

Nexo Revista Científica / Vol. 33, No. 01, pp. 36-50 / Junio 2020 
Se observa que el calentamiento de los aceites no afectó la densidad de los mismos, siendo los valores obtenidos los mismos para las muestras que se mantuvieron a temperatura ambiente $\left(26^{\circ} \mathrm{C}\right)$ y las que se sometieron a calentamiento a $140^{\circ} \mathrm{C}$. También se observó que los dos aceites presentan la misma densidad. En el caso de la viscosidad, se observó que al someter los aceites a calentamiento, esta propiedad aumenta, lo que indica que por efecto del calor, se llevan a cabo cambios en la estructura molecular de los aceites, lo que hace que ofrezcan mayor resistencia a fluir. Lo anterior se fundamenta en el hecho de que para que la viscosidad aumente se puede deber a dos factores, el primero a la vaporización y pérdida de los componentes más livianos de los aceites, sin embargo esto también debió influir en la densidad, lo cual no se observó, el otro efecto es que la temperatura promueva reacciones de polimerización y por lo tanto cambios en la estructura molecular, lo cual influye en el aumento de la viscosidad. Aunque no es objeto de estudio de la presente investigación, es claro que el someter los aceites a calentamiento provoca cambios en los mismos, lo que puede influir en el desempeño que estos tengas como estabilizantes de asfaltenos. Igualmente la viscosidad del aceite de $C$. nucifera es menor a la obtenida para el aceite de $J$. gossypiifolia.

En la tabla 3, se muestran las propiedades obtenidas para la muestra de petróleo curdo utilizado en la investigación.

Tabla 3. Propiedades de la muestra de petróleo crudo

\begin{tabular}{cccc}
\hline Propiedad & Valor & Unidad & Norma \\
\hline Gravedad API & 26.4 & ${ }^{\circ}$ API & ASTM D287 \\
Viscosidad & 56,8 & $\mathrm{cP}$ & ASTM D2196 \\
Saturados & 39,4 & $\%$ & \\
Aromáticos & 25,7 & $\%$ & \multirow{2}{*}{ ASTM D2007 } \\
Resinas & 30,4 & $\%$ & \\
Asfaltenos & 4,2 & $\%$ &
\end{tabular}

Se observa que la muestra de petróleo crudo tiene una gravedad API que lo clasifica como de grado mediano, según la clasificación establecida por la American Petroleum Institute (API), tomando como base lo que menciona The International Council on Clean Transportation (ICCT) [2011]. Esta clasificación coincide con los petróleos producidos en la zona de donde se obtuvo la muestra (Campo El Furrial), la cual se caracteriza por la producción de crudos entre 23 y $36^{\circ} \mathrm{API}$, con promedio de $27^{\circ} \mathrm{API}$ (Almarza, 1998). La composición SARA muestra predominancia de componentes Saturados, sin embargo también posee una cantidad importante de Resinas y Aromáticos, con menor cantidad de Asfaltenos. Sin embargo el porcentaje de asfaltenos de la muestra se puede considerar importante y representativo del campo petrolífero de donde se extrajo la muestra, cuyo rango se encuentra entre 1 y $6 \%$ (Almarza, 1998).

Con la composición SARA, se calculó el índice de estabilidad coloidal de asfaltenos según Asomaning y Watkinson (2000), el cual relaciona Aromáticos + Resinas y Saturados + Asfaltenos, como se muestra en la ecuación 3.

$$
I E C=\frac{\text { Saturados }+ \text { Asfaltenos }}{\text { Aromáticos }+ \text { Resinas }}
$$

El resultado fue de 0,78 lo que clasifica a la muestra como un crudo estable (IEC < 0,9), es decir que presenta poca tendencia a producir precipitación de asfaltenos, sin embargo al estar el valor cercano al límite es un indicativo de que la estabilidad se puede ver afectada por cambios en las condiciones termodinámicas a las que se someta el crudo.

Al determinar el INE original de la muestra de petróleo crudo, se obtuvo un valor de $0,81 \pm 0,01$, el cual fue tomado como referencia para el cálculo de la eficiencia porcentual de cada uno de los tratamientos aplicados.

Nexo Revista Científica / Vol. 33, No. 01, pp. 36-50 / Junio 2020 
En la tabla 4 se muestran los resultados obtenidos de luego del desarrollo del diseño experimental planteado y el cálculo de los INE y el porcentaje de eficiencia de cada uno de los tratamientos.

Tabla 4. Resultados de la aplicación de los tratamientos

\begin{tabular}{cccc}
\hline Tratamiento & INE & Desviación Estándar & $\%$ Ef \\
\hline D26 & 0,81 & 0,03 & 0,13 \\
D140 & 0,79 & 0,02 & 1,88 \\
AC2630 & 0,72 & 0,04 & 11,08 \\
AC2640 & 0,45 & 0,05 & 44,49 \\
AC2650 & 0,40 & 0,02 & 50,12 \\
AC14030 & 0,53 & 0,08 & 34,78 \\
AC14040 & 0,39 & 0,03 & 51,52 \\
AC14050 & 0,36 & 0,03 & 55,91 \\
AT2630 & 0,53 & 0,01 & 35,10 \\
AT2640 & 0,24 & 0,04 & 70,30 \\
AT2650 & 0,61 & 0,02 & 25,13 \\
AT14030 & 0,36 & 0,02 & 55,19 \\
AT14040 & 0,23 & 0,02 & 71,89 \\
AT14050 & 0,27 & 0,01 & 67,13 \\
\hline \multicolumn{2}{c}{ Fuente: Elaboración propia }
\end{tabular}

Las menores eficiencias se obtuvieron al aplicar los tratamientos con diésel puro (D26 y D140), lo que indica que la presencia de los aceites en las mezclas es causante de los valores más altos de eficiencia, por lo que se puede descartar la influencia del solvente en la mezcla y que los aceites se comportan como el componente activo en la estabilización de los asfaltenos. Ambos aceites aumentaron su eficiencia al ser sometidos a calentamiento, observándose el mayor valor para el tratamiento con aceite de J. gossypiifolia al $40 \%$ con $71,89 \%$. En promedio, el aceite de J. gossypiifolia mostró mayor eficiencia a ambas temperaturas, presentando un mejor comportamiento para $140{ }^{\circ} \mathrm{C}$ con $49,01 \%$ de eficiencia promedio. Lo anterior sugiere, que se deben someter a calentamiento los aceites antes de ser utilizados como estabilizadores de asfaltenos.

En la tabla 5 se muestra el resultado de aplicar el análisis de varianza multifactorial para la variable INE. La prueba se realiza para identificar si existe influencia estadísticamente significativa de los factores experimentales sobre la variable respuesta INE.

Tabla 5. Análisis de Varianza para INE - Suma de Cuadrados Tipo III

\begin{tabular}{|c|c|c|c|c|c|}
\hline Fuente & Suma de Cuadrados & Gl & Cuadrado Medio & Razón-F & Valor-P \\
\hline \multicolumn{6}{|c|}{ EFECTOS PRINCIPALES } \\
\hline A:Tipo de aceite & 0,06977 & 1 & 0,06977 & 60,12 & 0.0000 \\
\hline B:Porcentaje de aceite & 1,50919 & 3 & 0,50306 & 433,52 & 0.0000 \\
\hline C:Temperatura & 0,09100 & 1 & 0,09100 & 78,42 & 0.0000 \\
\hline \multicolumn{6}{|l|}{ INTERACCIONES } \\
\hline $\mathrm{AB}$ & 0,09977 & 3 & 0,03326 & 28,66 & 0.0000 \\
\hline $\mathrm{AC}$ & 0,01725 & 1 & 0,01725 & 14,87 & 0.0005 \\
\hline $\mathrm{BC}$ & 0,14937 & 3 & 0,04979 & 42,91 & 0.0000 \\
\hline $\mathrm{ABC}$ & 0,08686 & 3 & 0,02895 & 24,95 & 0.0000 \\
\hline RESIDUOS & 0,03713 & 32 & 0,00116 & & \\
\hline TOTAL (CORREGIDO) & 2,06035 & 47 & & & \\
\hline
\end{tabular}

Se observa en la tabla 5, que todos los resultados de Valor-P son menores a 0,05 , por lo que se concluye que los mismos tienen influencia estadísticamente significativa sobre los valores obtenidos para INE con un nivel de confianza de $95 \%$. No solo los factores influyen individualmente, sino que sus interacciones

Nexo Revista Científica / Vol. 33, No. 01, pp. 36-50 / Junio 2020 
también tienen influencia estadísticamente significativa. Es de especial importancia que la combinación de los tres factores muestre influencia significativa, lo que indica que no solo influye el tipo de aceite en combinación con su porcentaje en la mezcla, sino que también su efecto tiene dependencia con la temperatura a la cual se someta el aceite antes de ser mezclado y aplicado al petróleo.

Para observar la influencia de cada factor de manera individual, se realizó la prueba de rangos múltiples de Fisher (LSD) y los resultados se muestran en la tabla 6.

Tabla 6. Pruebas de Múltiple Rangos para INE

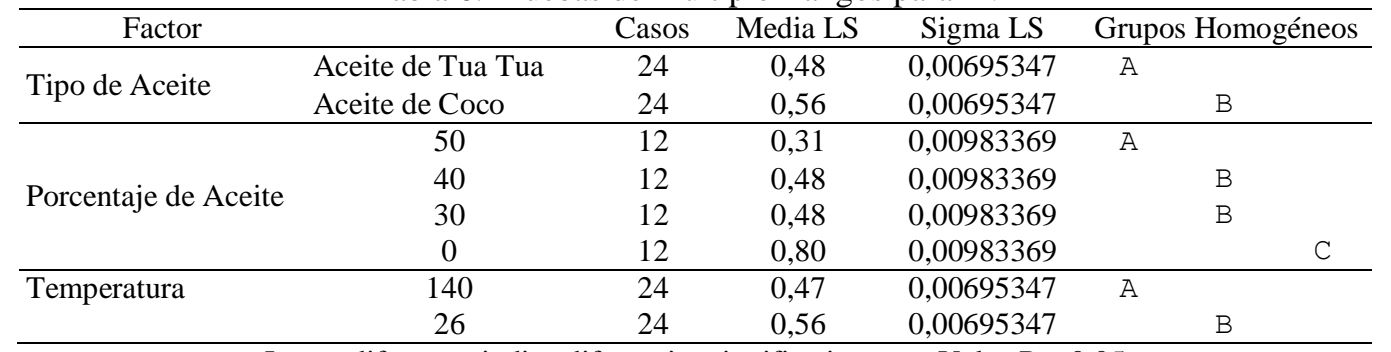

Letras diferentes indica diferencias significativas con Valor-P $<0,05$

Fuente: Elaboración propia a partir de resultados del paquete Statgraphics Centurion XVI.I.

Se observa que hay diferencias significativas (Valor-P $<0,05$ ) entre los tipos de aceite, lo que indica que la estabilidad de los asfaltenos en la muestra de petróleo utilizada depende del tipo de aceite utilizado, además se observa que los resultados favorecen al aceite de Tua tua (J. gossypiifolia) con el que se obtiene un menor INE. Así mismo el factor temperatura presenta diferencias significativas, corroborando que al someter los aceites a calentamiento, su efecto sobre INE se ve afectado, con un comportamiento que favorece a las muestras de aceites calentados a $140{ }^{\circ} \mathrm{C}$. El factor porcentaje de aceite, al igual que los otros dos anteriormente analizados, mostró diferencias significativas, sin embargo se observa que al aplicar los aceites a 30 y $40 \%$, no se obtienen resultados diferentes, es decir, que las diferencias entre los valores de INE no son estadísticamente significativos. La mezcla de aceite a $50 \%$ produjo resultados con diferencia significativa respecto a las otras dos mezclas, siendo éste porcentaje el que mostró el mejor desempeño al disminuir en mayor proporción el INE. Así mismo se observó que los tratamientos con las mezclas de aceite y diésel produjeron valores de INE estadísticamente diferentes a los obtenidos con la aplicación de 100\% diésel, lo que corrobora el efecto de la aplicación de los aceites evaluados.

La tendencia del comportamiento del INE respecto al porcentaje de aceite en la mezcla se observa en las figuras 2, 3, 4 y 5. Las curvas de tendencia se obtuvieron a partir de regresión no lineal intrínsecamente lineal.

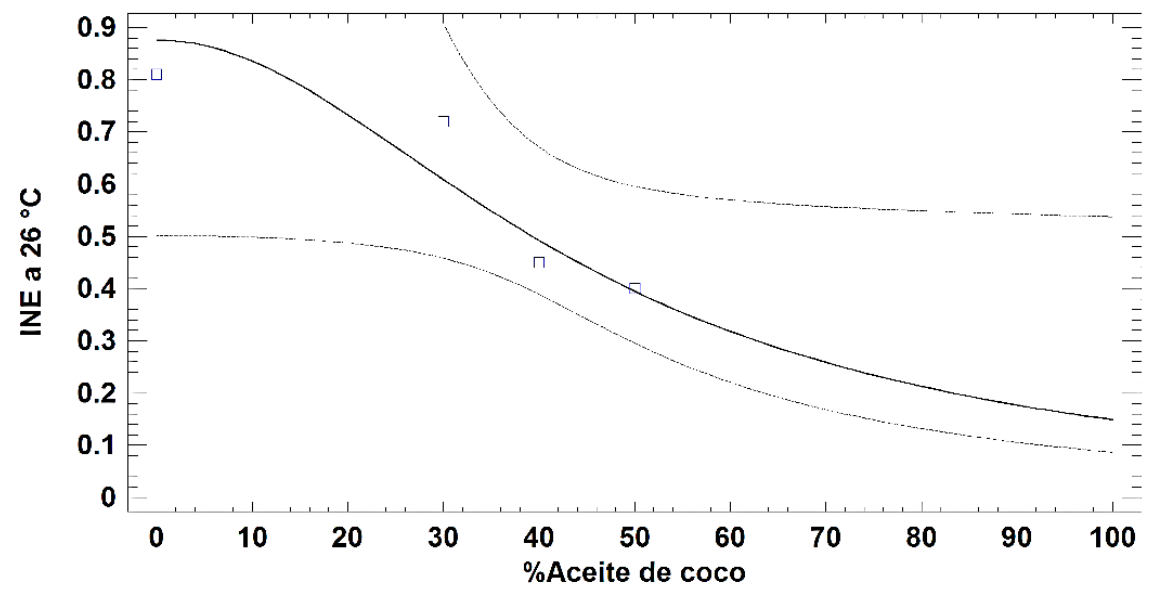

Figura 2. Representación gráfica del comportamiento del INE al aplicar aceite de coco a T $=26{ }^{\circ} \mathrm{C}$ Fuente: Elaboración propia

Nexo Revista Científica / Vol. 33, No. 01, pp. 36-50 / Junio 2020 
Se observa que el comportamiento de la relación entre los porcentajes de aceite de coco a $26{ }^{\circ} \mathrm{C}$, y los valores de INE es no lineal con un coeficiente de determinación $\mathrm{R}^{2}$ de 0,90 lo que indica que el modelo describe la variabilidad del INE de la muestra de crudo al aplicar aceite de coco en un $90,0 \%$. Además se puede estimar que la eficiencia máxima será cercana a 80,0\%. Adicionalmente se calculó el Valor-P del modelo, el cual fue menor a 0,05 lo que significa que el mismo es estadísticamente significativo.

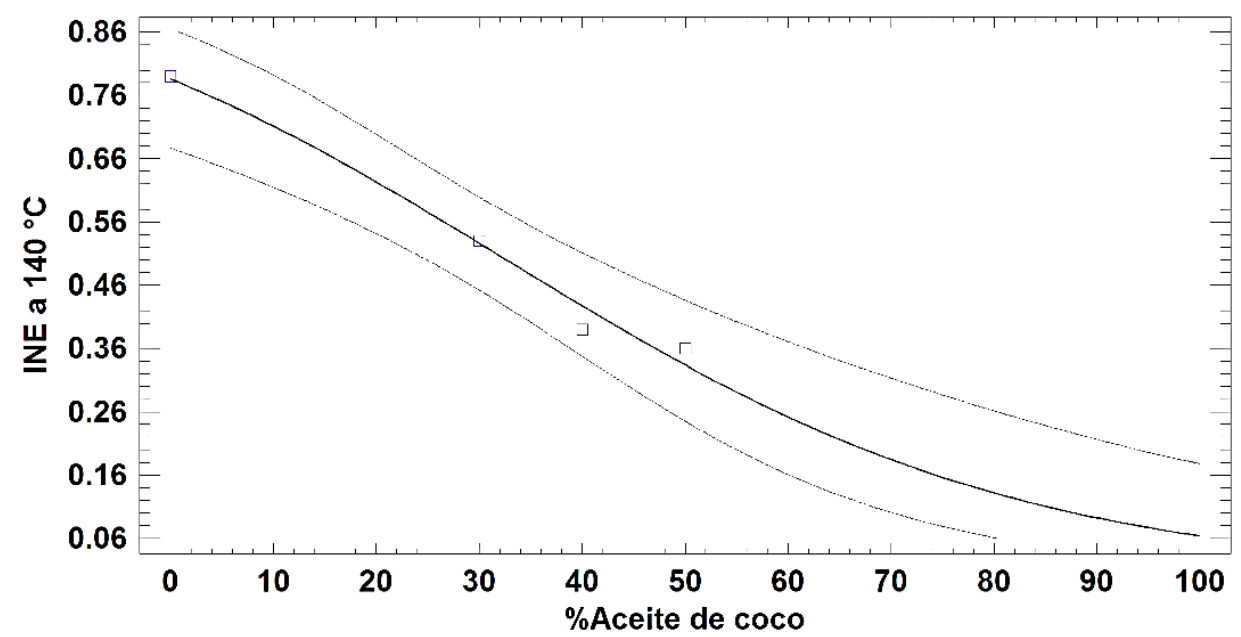

Figura 3. Representación gráfica del comportamiento del INE al aplicar aceite de coco a $\mathrm{T}=140{ }^{\circ} \mathrm{C}$ Fuente: Elaboración propia

El comportamiento gráfico del INE respecto al porcentaje de aceite de coco calentado a $140{ }^{\circ} \mathrm{C}$, en la mezcla arrojó un coeficiente de determinación $\mathrm{R}^{2}=0,983$ lo que indica que explica el 98,3\% de la variabilidad de INE cuando se aplican diferentes porcentajes de aceite en la mezcla con diésel. Con el modelo matemático se puede predecir que el máximo de eficiencia para el aceite calentado a $140{ }^{\circ} \mathrm{C}$ se estima en $99,5 \%$. Adicionalmente se calculó el Valor-P del modelo, el cual fue menor a 0,05 lo que significa que el mismo es estadísticamente significativo.

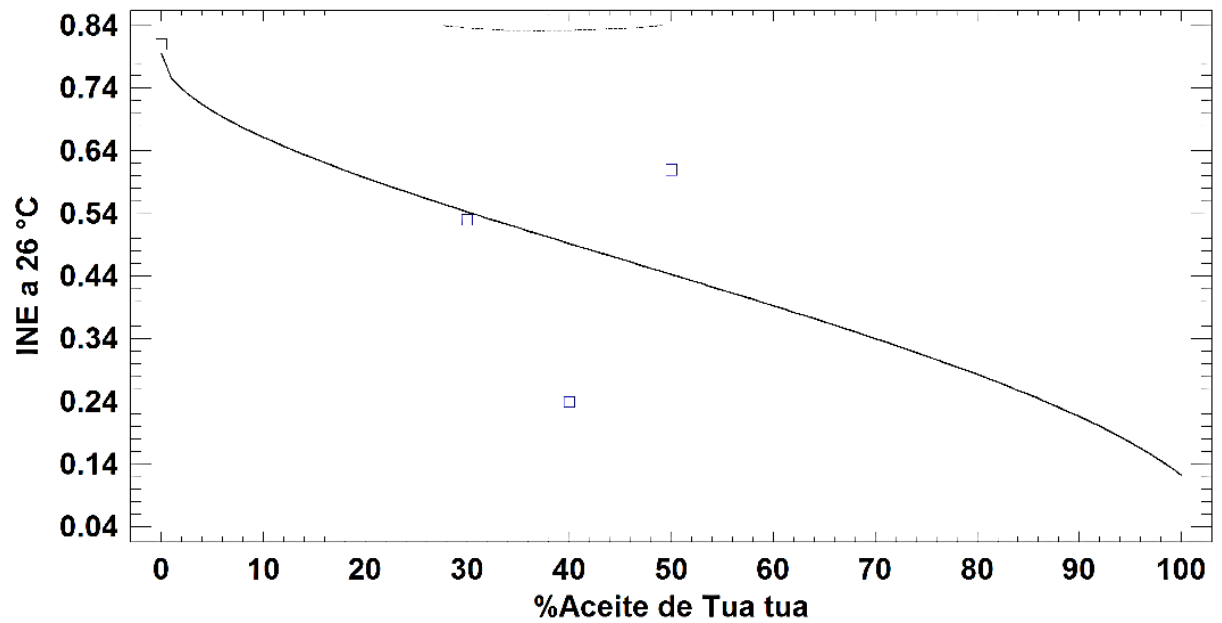

Figura 4. Representación gráfica del comportamiento del INE al aplicar aceite de Tua tua a $\mathrm{T}=26{ }^{\circ} \mathrm{C}$ Fuente: Elaboración propia

Al graficar los valores de INE respecto a los porcentajes de aceite de Tua tua, a $26{ }^{\circ} \mathrm{C}$ se observó que la tendencia de los mismo también se ajustan a un modelo no lineal, sin embargo el Valor-P fue mayor a 0,05 $(0,198)$, lo que indica que el modelo no es estadísticamente significativo (Figura 4). Además se obtuvo un

Nexo Revista Científica / Vol. 33, No. 01, pp. 36-50 / Junio 2020 
$\mathrm{R}^{2}=0,643$ por lo que la variabilidad de los valores de INE son explicados en $64,3 \%$ por la porcentajes de aceite presente en la mezcla. Debido a la poca significancia del modelo no es posible estimar un valor máximo de eficiencia.

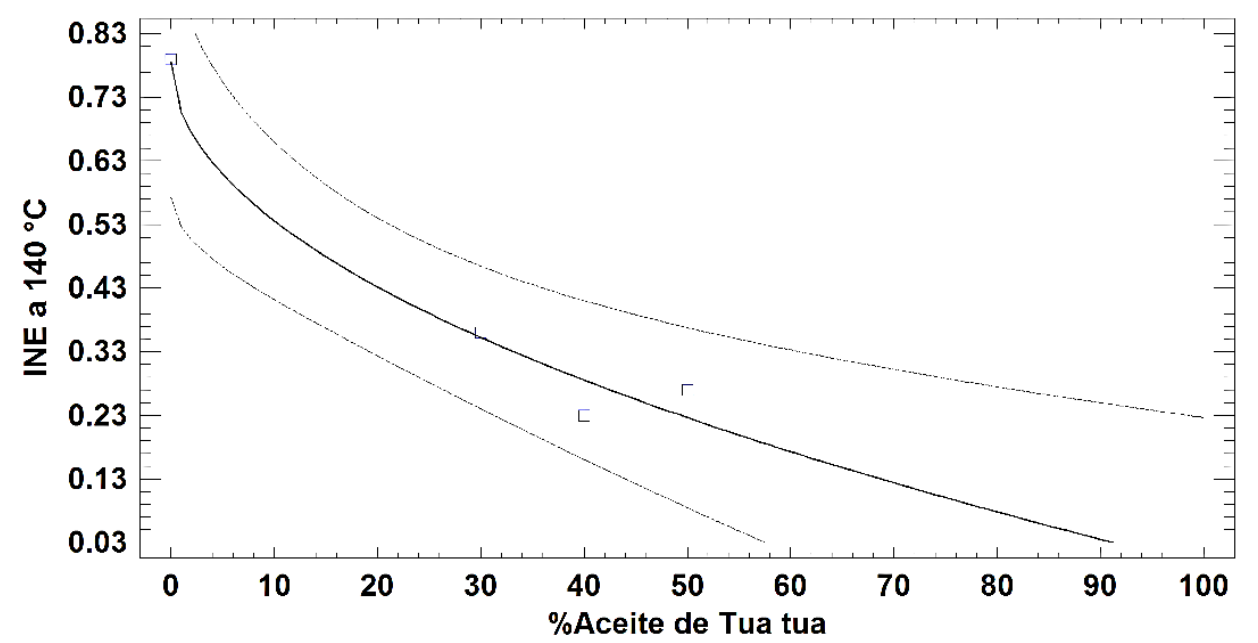

Figura 5. Representación gráfica del comportamiento del INE al aplicar aceite de Tua tua a $\mathrm{T}=140{ }^{\circ} \mathrm{C}$

El modelo matemático para la relación entre INE y los porcentajes de aceite de Tua tua calentado a una temperatura de $140{ }^{\circ} \mathrm{C}$, mostró una tendencia no lineal y significativa (Valor-P =0,01). El coeficiente de determinación obtenido fue $\mathrm{R}^{2}=0,98$ por lo que el mismo explica la variabilidad de INE en un $98,0 \%$ al utilizar diferentes porcentajes de aceite en la mezcla. La eficiencia máxima estimada a través de la tendencia obtenida es de $99,8 \%$.

\section{DISCUSIÓN}

Las características obtenidas de los aceites demostraron que ambos presentan una densidad similar, la cual no varía aun cuando se sometan los mismos a calentamiento. En el caso de la J. gossypiifolia, la densidad obtenida de 0,92 g/ml coincide con la reportada por De Oliveira et al. (2009), sin embargo al ser un aceite poco estudiado no se encuentra en la literatura un valor de viscosidad con el cual comparar. El aceite de $C$. nucifera, también reportó el mismo valor de densidad, el cual fue mayor al reportado por Marín (2019) quien obtuvo entre 0,86 y $0,89 \mathrm{~g} / \mathrm{ml}$, pero coinciden con la densidad reportada por Bello, Adekanbi y Akinbode (2015) quienes obtuvieron un valor de $0,925 \mathrm{~g} / \mathrm{ml}$. La viscosidad del aceite de $C$. nucifera fue reportada en $19,34 \mathrm{cP}$ a $25{ }^{\circ} \mathrm{C}$ y $19,38 \mathrm{cP}$ a1 $40{ }^{\circ} \mathrm{C}$ por Marín (2019), valores que no coinciden con los obtenidos en la presente investigación, aun cuando se observa la misma tendencia al aumento respecto a la temperatura. Bello et al. (2015) reportaron una viscosidad de $27,23 \mathrm{cP}$ a $40{ }^{\circ} \mathrm{C}$, valor más ajustado a la tendencia observada. Las diferencias entre las propiedades del aceite están influenciadas por la variedad de coco utilizada y el método de extracción aplicado, lo que pudo influir en las diferencias observadas respecto a las otras investigaciones.

Las propiedades de la muestra de petróleo utilizada en la investigación, corresponden en promedio a las producidas en el Campo El Furrial del Estado Monagas, Venezuela, lo que concuerda con lo que manifestado por Beltrán, (2010). Aunque no se puede generalizar con la composición SARA del petróleo, la tendencia observada guarda cierto grado de coincidencia con los valores reportados por Lamus et al. (2011) quien analizó petróleos crudos medianos de Colombia, obteniendo valores de Saturados entre 27 y $54 \%$, Aromáticos entre 21 y $35 \%$, Resinas entre 5 y $11 \%$ y Asfaltenos entre 1 y $9 \%$. Se observó que los valores obtenidos en la presente investigación se encuentran en los rangos mostrados, a excepción de las

Nexo Revista Científica / Vol. 33, No. 01, pp. 36-50 / Junio 2020 
resinas, que estuvieron por encima del rango, sin embargo la composición del petróleo varía de una zona productora a otra, por lo que no se puede generalizar respecto a estos resultados.

Los resultados obtenidos del INE al aplicar cada uno de los tratamientos, demuestran que los dos aceites evaluados tienen efecto sobre la estabilidad de los asfaltenos en la muestra de petróleo utilizada, llegando a mejorar la misma. En el caso del aceite de coco, su eficiencia ha sido reportada en investigaciones como la de Bello et al. (2015) quienes concluyeron que la mezcla de aceite de $C$. nucifera y diésel, muestra eficiencias que superaron las obtenidas al utilizar productos comerciales y las mismas llegaron a valores entre 60,0 y $97,2 \%$ al ser aplicados en dosis de $8 \mu \mathrm{l}$ a dos diferentes crudos medianos de campos productores del Estado Monagas, Venezuela. Lo anterior es consistente con lo obtenido en la presente investigación, sin embargo la máxima eficiencia alcanzada de 55,9\% es menor a las reportadas en la citada investigación, lo que se puede deber a diferencias en la composición propia de cada crudo, ya que el aceite no fue aplicado a la misma muestra. Así mismo, Marín (2019) también demostró que la temperatura a la cual se somete el aceite de $C$. nucifera antes de ser mezclado con el diésel influye de manera significativa en su desempeño como estabilizante de asfaltenos, lo que es coincidente con lo observado en la presente investigación, sin embargo el autor citado establece una temperatura de máxima eficiencia de $130{ }^{\circ} \mathrm{C}$, la cual es menor a la utilizada, pero igual se utilizaron muestras de petróleo diferentes en ambas investigaciones, por lo que no se puede generalizar sobre este factor. Marín (2019) reporta a la temperatura de $130{ }^{\circ} \mathrm{C}$ una eficiencia de $50,0 \%$ lo que coincide con lo observado, siendo este valor aún menor al obtenido en la presente investigación.

El aceite de la semilla de $J$. gossypiifolia no ha sido estudiado como estabilizante de asfaltenos, sin embargo el de otra planta de la misma familia, la Jatropha curcas, fue utilizado por Marín et al. (2016) quienes demostraron la aplicabilidad de dicho aceite mezclado con diésel, llagando a obtener eficiencias de hasta $88,8 \%$, la cual es mayor al 71,9\% máximo observado en la presente investigación, sin embargo no se pueden comparar ambos aceites debido a sus diferentes composiciones, las cuales fueron reportadas por De Oliveira et al. (2009), siendo el componente mayoritario del aceite de J. gossypiifolia, el ácido oleico (C18:1) y para el aceite de J. curcas, el ácido linoleico (C18:2), también los autores citados reportan que la J.gossypiifolia contiene una mayor proporción de ácido palmítico (C16:0) que la J. curcas, resultados que son corroborados por Ceasar y Ignacimuthu (2011) y Sousa et al. (2015). Los resultados obtenidos demuestran que el aceite de $J$. gossypiifolia mezclado con diésel tiene propiedades estabilizante de asfaltenos, llagando a superar en eficiencia al aceite de $C$. nucifera, por lo que su uso puede llegar a ser de gran importancia, sobre todo por ser una planta que se distribuye ampliamente en América y África, además de que su aceite no es comestible como es el caso del aceite de coco, lo que le daría un uso diferente a la planta, además de los usos medicinales actuales (Wu et al. 2019). Lo anterior es corroborado por el análisis estadístico realizado, en donde se observó diferencias significativas con un nivel de confianza de 95\%, al comparar ambos aceites, las cuales fueron favorables al de J. gossypiifolia.

Se observó que los aceites vegetales utilizados son potenciales sustitutos de productos comerciales sintéticos para el tratamiento de la precipitación de los asfaltenos en el petróleo, lo que coincide con investigaciones anteriores (Safaie y Naza, 2014; Bello et al., 2015; Marín et al., 2016; Afra et al., 2018; Marín, 2019), lo que representa un amplio campo de investigación para el futuro, en la búsqueda de encontrar alternativas eficientes, sostenibles, ecoamigables y económicas para un problema que se estará presentando siempre en la producción petrolera, debido a que los asfaltenos son componentes presentes en la mayoría de los petróleos que se producen a nivel mundial.

\section{CONCLUSIONES}

Los aceites vegetales evaluados demostraron que tienen actividad estabilizante de los asfaltenos presentes en el petróleo crudo proveniente del Campo El Furrial en el Estado Monagas Venezuela, al disminuir el Índice de Inestabilidad del mismo. 
El análisis estadístico indica que el desempeño de los dos aceites evaluados como estabilizantes de asfaltenos, presenta diferencias significativas con un nivel de confianza de 95\%, siendo el aceite de $J$. gossypiifolia el que mostró mayor eficiencia al ser aplicada a la muestra de petróleo crudo con 71,89\%.

El factor temperatura también tuvo influencia significativa sobre la eficiencia de los aceites evaluados, concluyéndose que para las condiciones de la investigación, al aplicar calentamiento a los mismos a $140{ }^{\circ} \mathrm{C}$ por $24 \mathrm{~h}$, se produce un aumento en la eficiencia estabilizadora.

El factor porcentaje de aceite también presentó influencia estadística significativa en la estabilidad de los asfaltenos, siendo la mezcla más eficiente fue la de 50\% aceite en diésel, para las dos muestras evaluadas, así mismo, la combinación de los tres factores (Temperatura, Tipo de Aceite y Porcentaje de aceite) influye sobre la estabilidad.

De los dos aceites evaluados, el obtenido de las semillas de la J. gossypiifolia representa una alternativa factible para ser utilizado como estabilizante de asfaltenos, por no ser comestible y proveniente de una planta que crece de manera silvestre en una zona geográfica amplia, por lo que se debe seguir investigando sobre la factibilidad de producirlo de manera comercial, lo que amerita estudios agronómicos además de factibilidad de producción y aplicación del aceite a muestras de petróleo diferentes y bajo condiciones específicas similares a las de operación.

\section{REFERENCIAS}

Almarza, R. (1998). Campos Petrolíferos de Venezuela. Acta Geológica Venezolana. Pdvsa-Intevep. Recuperado de http://www.pdvsa.com/lexico/camposp/cp051.htm

Afra, S., Nasr-El-Din, H.A., Socci, D. y Cui, Z. (2018). Green phenolic amphiphile as a viscosity modifier and asphaltenes dispersant for heavy and extra-heavy oil. Fuel, 220, 481-489.

Akbarzadeh, K., Allenson, S., Creek, J. y Jamaluddin, A. (2007). Asphaltenes Problematic but Rich in Potential. Oilfield Review, 9(2), 22-48.

Asomaning, S. y Watkinson, A.P. (2000). Petroleum Stability and Heteroatom Species Effects in Fouling of Heat Exchangers by Asphaltenes. Heat Transfer Engineering, 21(3), 10-16.

ASTM D287. (2012). Standard Test Method for API Gravity of Crude Petroleum and Petroleum Products (Hydrometer Method). West Conshohocken, PA: American Society for Testing and Materials.

ASTM D1298. (2017). Standard Test Method for Density, Relative Density, or API Gravity of Crude Petroleum and Liquid Petroleum Products by Hydrometer Method. West Conshohocken, PA: American Society for Testing and Materials.

ASTM D2007. (2011). Standard Test Method for Characteristic Groups in Rubber Extender and Processing Oils and Other Petroleum-Derived Oils by the Clay-Gel Absorption Chromatographic Method. West Conshohocken, PA: American Society for Testing and Materials.

ASTM D2196. (2015). Standard Test Methods for Rheological Properties of Non-Newtonian Materials by Rotational Viscometer. West Conshohocken, PA: American Society for Testing and Materials.

Bello, E.I., Adekanbi, I.T. y Akinbode, F.O. (2015). Production and characterization of coconut (Cocos nucifera) oil and its methyl ester. European Journal of Engineering and Technology, 3(3), 25-35. 
Bello, Y.B., Manzano, J.R. y Marín, T.D. (2015). Análisis comparativo de la eficiencia dispersora de asfaltenos de productos a base de aceite de coco (Cocus nucifera) como componente activo y dispersantes comerciales aplicados a muestras de petróleo del Campo el Furrial, Estado Monagas, Venezuela. Revista Tecnológica ESPOL - RTE, 28(2), 51-61.

Ceasar, S.A. y Ignacimuthu, S. (2011). Applications of biotechnology and biochemical engineering for the improvement of Jatropha and Biodiesel: A review. Renewable and Sustainable Energy Reviews, 15, 51765185.

Beltrán, O.J. (2010). Caracterización petrofísica y sedimentológica mediante el análisis de núcleos en yacimientos de la formación Naricual, cuenca oriental de Venezuela (Tesis de maestría). Maracaibo, Venezuela: Universidad Del Zulia.

Da Silva, C.P., Restrepo, F.I., Peralta, I.A., Vasquez, M.M., Portura, M.L. y Arango, Y. (2017). Extracción de aceite de Coco (Cocos nucifera) como estrategias de aprovechamiento de los productos locales de Mitú. Recuperado de: http://revistas.sena.edu.co/index.php/rvin/article/view/688/759

De Almeida, P.M., De Sousa, S., Marin-Morales, M.A., Benko-Iseppon, A.M. y Brasileiro-Vidal, A.C. (2015). Genotoxic potential of the latex from cotton-leaf physicnut (Jatropha gossypiifolia L.). Genetics and Molecular Biology, 38(1), 93-100.

De Oliveira, J.S., Leite, P.M., De Souza, L.B., Mello, V.M., Silva, E.C., Rubim, J.C., Meneghetti, S.M.P. y Suarez, P.A.Z. (2009). Characteristics and composition of Jatropha gossypiifolia and Jatropha curcas L. oils and application for biodiesel production. Biomass and bioenergy, 33, 449-453.

Félix-Silva, J., Brandt, R., Da Silva-Jr, A.A., Zucolotto, S.M. y Fernandes-Pedrosa, M. (2014). Jatropha gossypiifolia L. (Euphorbiaceae): A Review of Traditional Uses, Phytochemistry, Pharmacology, and Toxicology of This Medicinal Plant. Recuperado de: http://dx.doi.org/10.1155/2014/369204

Ghaffar, A.M.A., Kabel, K.I., Farag, R.K., Maysour, N.E. y Zahran, M.A.H. (2013). Synthesis of poly (dodecyl phenol formaldehyde)-b-poly (oxypropylene) block copolymer, and evaluation as asphaltene inhibitor and dispersant. Research on Chemical Intermediates, 41(1), 443-455. doi: 10.1007/s11164-013$1243-8$

Goual, L. (2012). Petroleum Asphaltenes. En Crude Oil Emulsions - Composition Stability and Characterization. USA: InTech.

ICCT. (2011). Introducción a la refinación del petróleo y producción de gasolina y diésel con contenido ultra bajo de azufre. Recuperado de https://theicct.org/sites/default/files/ICCT_RefiningTutorial_Spanish.pdf.

Islam, M., Islam, A. y Hossain, M.S. (2017). Biological Investigation of Jatropha gossypiifolia: A Stiff Medicinal Plant in Bangladesh. Iranian Journal of Pharmaceutical Sciences, 13(1), 35-48.

Lafont, J.J., Páez, M.S. y Portacio, A.A. (2011). Extracción y Caracterización Fisicoquímica del Aceite de la Semilla (Almendra) del Marañón (Anacardium occidentale L). Información Tecnológica, 22(1), 51-58.

Lamus, C., Guzmán, A., Murcia, B., Cabanzo, R. y Mejía-Ospino, E. (2011). Uso de Análisis Multivariado En La Determinación SARA De Crudos Por Espectroscopia NIR. Revista Colombiana de Física, 43(3), 635-64. 
Madh, M., Kharrat, R. y Hamoule, T. (2017). Screening of inhibitors for remediation of asphaltene deposits: Experimental and modeling study. Petroleum. Recuperado de: http://doi.org/10.1016/j.petlm.2017.08.001.

Mansoori, G. (2009). A unified perspective on the phase behaviour of petroleum fluids. International Journal Oil, Gas and Coal Technology, 2(2), 141-167.

Marín, T., Marcano, S. y Febres, M. (2016). Evaluación del aceite de Jatropha curcas como aditivo dispersante de asfaltenos en un crudo del campo el Furrial, Venezuela. Ingeniería-Revista Académica de la Facultad de Ingeniería, Universidad Autónoma de Yucatán, 20(2), 98-107.

Marín, T.D. (2019). El aceite de coco (Cocos nucifera) como estabilizante de asfaltenos en un crudo del Estado Monagas, Venezuela: efecto de la temperatura. Ingeniería y Desarrollo, 37(2), 289-305.

Mehrotra, A.K. y Bidmus, H.O. (2004). Heat-Transfer Calculations for Predicting Solids Deposition in Pipeline Transportation of "Waxy" Crude Oils. Calgary, Canada: University of Calgary.

Oyedeji, K.O., Akinbiyi, P., Oni, J. y Obisesan, A. (2018). Visceral Histopathological Alterations in Rats Treated with Ethanol Extract of Jatropha gossypifolia (POHL). Journal of Pharmaceutical Science and Research, 10(5), 1027-1029.

Pereira, J.C., Delgado-Linares, J., Briones, A., Guevara, M., Scorzza, C. y Salager, J.L. (2011). The Effect of Solvent Nature and Dispersant Performance on Asphaltene Precipitation from Diluted Solutions of Instable Crude Oil. Petroleum Science and Technology, 29, 2432-2440.

Pineda-Flores, G. y Mesta-Howard, A. (2001). Petroleum asphaltenes: generated problematic and possible biodegradation mechanisms. Revista Latinoamericana de Microbiología, 43(3), 143-150.

Rezaee, E., Heidarizadeh, F., Sajjadifar, S. y Abbasi, Z. (2013). Dispersing of Petroleum Asphaltenes by Acidic Ionic Liquid and Determination by UV-Visible Spectroscopy. Recuperado de: https://doi.org/10.1155/2013/203036

Saeedi, A.H. y Hasan, M. (2017). Inhibiting asphaltene precipitation from Iranian crude oil using various dispersants: Experimental investigation through viscometry and thermodynamic modelling. Fluid Phase Equilibria, 442, 104-118

Safaie, K. y Naza, A. (2014). Evaluation of Asphaltene Inhibitors Effect on Aggregation Coupled Sedimentation Process. Journal of Dispersion Science and Technology, 35(3), 329-337.

Sepúlveda, J., Bonilla, J. y Medina, Y. (2010). Predicción de la Estabilidad de los Asfaltenos mediante la utilización del análisis SARA para Petróleos puros. Revista Ingeniería y Región, 7(1), 103-110.

Sousa, T.F., Castro, N.H., Queiroz, M.F., Fernandes, P.D., Mendonça, S., Aquino, J.A. y Medeiros, E.P. (2015). Fatty acid profiles of species of Jatropha curcas L., Jatropha mollissima (Pohl) Baill. and Jatropha gossypiifolia L. Industrial Crops and Products, 73, 106-108.

Spiecker P.M., Gawrys, K.L. y Kilpatrick, P.K. (2003). Aggregation and solubility behavior of asphaltenes and their subfractions. Journal of Colloid and Interface Science, 267, 178-193.

Wu, Q., Patocka, J., Nepovimova, E. y Kuca, K. (2019). Jatropha gossypiifolia L. and its biologically active metabolites: A mini review. Journal of Ethnopharmacology, 234, 197-203.

Nexo Revista Científica / Vol. 33, No. 01, pp. 36-50 / Junio 2020 
Yaro, A.H., Aliyu, M., Garba, K. y Hassan, S. (2018). Anti-seizure activity of Extract of Jatropha gossypiifolia Linn (Euphorbiaceae). Tropical Journal of Natural Product Research, 2(2), 99-102.

\section{SEMBLANZA DE LOS AUTORES}

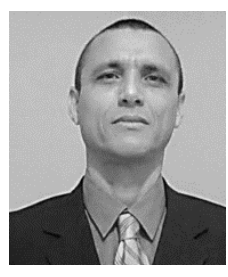

Tomás D. Marín-Velásquez: Obtuvo el grado de Ingeniero Químico en la Universidad de Oriente, Venezuela, donde fue profesor agregado, investigador, Director de Escuela de Ingeniería, Coordinador de Trabajos de Grado y miembro del comité editor de la revista científica PetroCiencias. Actualmente se desempeña como investigador en la empresa Innova Scientific, en Lima, Perú. Desarrolló sus estudios de maestría en la Universidad Internacional Iberoamericana, Puerto Rico, en Gestión y Auditorías Ambientales con mención en Ingeniería y Tecnología Ambiental. Ha trabajado en líneas de investigación vinculadas con el desarrollo de productos alternativos para el Tratamiento del Petróleo y para formular fluidos de perforación petrolera. También ha realizado investigación en Tratamiento de suelos y aguas contaminadas con hidrocarburos.

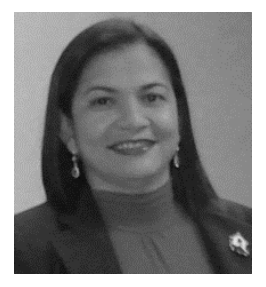

Dany Day J. Arriojas Tocuyo Obtuvo el grado de Ingeniero de Petróleo en la Universidad de Oriente, Venezuela. Se desempeñó como analista de calidad de datos en la empresa Petróleos de Venezuela (PDVSA). Actualmente se desempeña como investigador en la empresa Innova Scientific, en Lima, Perú. Desarrolló sus estudios de maestría en la Caribbean Internacional University, Curazao, en Administración de la Industria de los Hidrocarburos. Ha trabajado en líneas de investigación vinculadas con el desarrollo de productos alternativos para el Tratamiento del Petróleo, así como manejo integrado de datos de producción petrolera. También ha realizado investigación en Tratamiento de suelos y aguas contaminadas con hidrocarburos.

Nexo Revista Científica / Vol. 33, No. 01, pp. 36-50 / Junio 2020 